\title{
miR-508-5p acts as an anti-oncogene by targeting MESDC1 in hepatocellular carcinoma
}

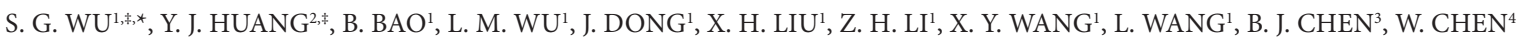 \\ ${ }^{1}$ Department of Clinical Laboratory, Guangzhou No. 12 Hospital, Guangzhou Medical University; ${ }^{2}$ Department of Clinical Laboratory, Guangzhou \\ Women and Children's Medical Center, Guangzhou Medical University; ${ }^{3}$ Department of Oncology and ${ }^{4}$ Department of General Surgery, Guangzhou \\ No. 12 Hospital, Guangzhou Medical University. \\ ${ }^{*}$ Correspondence: wsg0930@163.com \\ *Contributed equally to this work.
}

Received April 6, 2016 / Accepted August 12, 2016

\begin{abstract}
Hepatocellular carcinoma (HCC) is the third leading cause of cancer associated mortality. Accumulating evidence has shown that microRNAs (miRNAs) act as critical factors for tumor recurrence and metastasis. MiR-508-5p has been reported as a down-regulated miRNA in the primary gastric cancer tissues. However, the role of miR-508-5p on HCC has not been well elucidated. In this study, we observed that miR-508-5p was downregulated in HCC tissues when compared to the non-tumorous tissues. We then demonstrated that overexpression of miR-508-5p attenuated HepG2 cells proliferation and invasion and induced cell apoptosis in vitro. Furthermore, our further investigations revealed that mesoderm development candidate 1 (MESDC1) is a potential target of miR-508-5p, as well as miR-508-5p overexpression downregulated MESDC1 expression. Overexpression of MESDC1 promoted HepG2 cells migration, invasion and proliferation in vitro. In addition, miR-508-5p markedly suppressed the tumor growth in xenograft model, while MESDC1 promoted the tumor growth in xenograft model. This study provides new insight into molecular mechanisms that miR-508-5p acts as a tumor suppressor by targeting MESDC1 in HCC progression.
\end{abstract}

Key words: miR-508-5p, MESDC1, hepatocellular carcinoma

Hepatocellular carcinoma (HCC) is the fifth most frequent tumor and the third leading cause of cancer-related death worldwide [1]. Its incidence has increased dramatically with the most important causes, including viral (chronic hepatitis B and hepatitis C), toxic (alcohol), metabolic (diabetes and non-alcoholic fatty liver disease) and immune-related (primary biliary cirrhosis and autoimmune hepatitis) [2-4]. HCC has a 1-year survival rate after the onset of symptoms, and reliable tools for early diagnosis and treatment are lacking [5]. Therefore, there is an urgent need to explore early detection markers and novel therapeutic targets for the treatment of HCC.

MicroRNAs (miRNAs) are described as a class of endogenous 19-25 nucleotides non-coding RNAs that post-transcriptionally regulate gene expression $[1,6]$. It is now well known that miRNAs promote target mRNA degradation and inhibit mRNA translation by direct binding to the 3 '-untraslated region (3'-UTR) of mRNAs in diverse biological and pathological processes in life [7-9]. Accumulating evidence has demonstrated that numerous miRNAs were detected in specific tumor types and dysregulation of miRNAs expression was correlated with initiation, progression, metastasis, and chemo-resistance of tumors $[10,11]$. MiRNAs could act as either tumor suppressors or promoters in the development of various cancers. For example, miR-615 was identified as a tumor suppressor in breast cancer [12], whereas miR-31 has been showed to exert oncogenic functions in esophageal squamous cell carcinoma [13]. There are also many miRNAs were identified associated with development of HCC, including miR-1 [14], miR-129 [15] and miR-485-5p [16]. MiR-508-5p has been reported as a down-regulated miRNA in the primary gastric cancer tissues [17]. Y Shang et al [18] demonstrated that overexpression of miR-508-5p reversed drug resistance most efficiently in multiple resistance (MDR) gastric cancer by targeting ATPbinding cassette subfamily $\mathrm{B}$ member 1 (ABCB1) and Zinc ribbon domain-containing 1 (ZNRD1). These findings suggest 
that miR-508-5p is closely associated with the progression of tumors by specifically binding the seed sequence of target genes. Moreover, the underlying molecular mechanisms of miR-508$5 \mathrm{p}$ involved in HCC are not fully understood.

Mesoderm development candidate 1 (MESDC1) is emerging as a novel actin-binding protein located on chromosome $15 q 13$ [19]. However, the functional researches of MESDC1 in maintaining human physiological activities have been reported less. Tatarano et al [19] proved that MESDC1 protein has an oncogenic function in bladder cancer (BC) cell lines by inducing cell viability, migration and invasion and suppressing apoptosis. This functional study suggests that MESDC1 function as carcinogenic gene in tumor progression. However, it is still poorly whether MESDC1 was regulated by miRNA, and whether the association between miRNA and MESDC1 contributes to the progression of HCC.

In this study, we demonstrated that MESDC1 was a potential target of miR-508-5p according to bioinformatics analysis and results of luciferase reporter assay. Moreover, we found that miR-508-5p was downregulated in hepatocellular carcinoma tissues. We identified that overexpression of MESDC1 could significantly induce hepatoma cells migration, invasion, proliferation, and reduce cell apoptosis in vitro. Furthermore, overexpression of miR-508-5p in HepG2 cells markedly reduced MESDC1 expression, which resulted in suppression of cells invasion, migration, proliferation and promotion of cells apoptosis in vitro. In addition, miR-508$5 \mathrm{p}$ markedly suppressed growth of xenograft tumors in nude mice, while MESDC1 promoted growth of xenograft tumors in nude mice.

\section{Materials and methods}

Sources of tissue samples. Paired HCC and adjacent non-tumor liver tissues were obtained from 40 HCC patients undergoing HCC resection. The specimens were collected between June 2014 and June 2015 in the Department of Oncology as well as general surgery of No.12 Hospital. All tissues were confirmed by pathologic examination. Informed consent was obtained from each patient and ethical approval was obtained from the Institutional Review Board of Guangzhou No.12 Hospital, Guangzhou Medical University.

Cell culture. Human HepG2 cells were obtained from the American Type Culture Collection (ATCC; Manassas, VA, USA) and grown in Dulbecco's modified eagle's medium (DMEM) containing 10\% fetal calf serum (FCS) with streptomycin $(100 \mu \mathrm{g} / \mathrm{ml})$ and penicillin $(100 \mu \mathrm{g} / \mathrm{ml})$. HepG2 cells were incubated at $37^{\circ} \mathrm{C}$ in $5 \% \mathrm{CO}_{2}$. Cells were seeded in 6 - or 12 -well plates or $60-\mathrm{mm}$ dishes and grown to $80-90 \%$ confluency before use.

Lentivirus (LV) production and infection. Cells were cultured in $25 \mathrm{~cm}^{2}$ vented flasks containing DMEM with $10 \%$ FCS under standard culture conditions $\left(37^{\circ} \mathrm{C}\right.$ at $\left.5 \% \mathrm{CO} 2\right)$. Packed empty LV vectors with green fluorescent protein (GFP, LV-Mock), LV-mediated human miR-508-5p overexpression vector (LV-miR-508-5p), and LV-mediated MESDC1 overexpression vector (LV-MESDC1) with GFP were prepared as previously reported [20]. The cells were infected with the LV stock at a multiplicity of infection of 20 transducing units per cell in the presence of $8 \mathrm{mg} / \mathrm{ml}$ of polybrene. Then, cells were washed with fresh complete media after $24 \mathrm{~h}$ incubation. The GFP-positive cells were counted $96 \mathrm{~h}$ post-transduction.

RNA isolation and real-time quantitative PCR analysis. Total RNA from liver tissues was extracted using TRIzol reagent (Invitrogen, Carlsbad, CA, USA) in accordance with the manufacturer's instructions. The miRNA levels were determined by real-time quantitative PCR using an ABI 7500 Fast Real-Time PCR system with the All-in-One ${ }^{\mathrm{rm}}$ miRNA qPCR kit in $20 \mu \mathrm{l}$ reaction volumes (GeneCopoeia, Rockville, MD, USA) according to the instruction manual. The expression of U6 RNA was used as an endogenous control. Quantitative measurements were determined using the $\Delta \Delta \mathrm{Ct}$ method. The mRNA levels were determined by real-time quantitative PCR using an ABI 7500 Fast Real-Time PCR system with SYBRGreen Detection chemistry (Takara Bio, Inc., Shiga, Japan). The expression of GAPDH was used as the internal control. All samples were measured in triplicate and the mean value was considered for comparative analysis.

Transfection of miRNA mimics and small interfering RNA. HepG2 cells were transfected with $50 \mathrm{nM}$ miR-508-5p mimics utilizing Lipofectamine 2000 transfection reagent (Invitrogen) for $48 \mathrm{~h}$ according to the manufacturer's instructions. All experimental control samples were treated with an equal concentration of a non-targeting control mimic sequence (negative controls). The small interfering RNAs (siRNAs) against MESDC1 and an irrelevant 21-nucleotide control siRNA (Negative Control) were purchased from Ribo Biotechnology. Cells $\left(2 \times 10^{6} /\right.$ well $)$ were transfected using Lipofectamine 2000 transfection reagent for $48 \mathrm{~h}$ according to the manufacturer's instructions. After $48 \mathrm{~h}$ of transfection, RT-PCR and Western blot were performed.

Luciferase assay. Human MESDC1 cDNA, containing putative and mutant target sites for miR-508-5p, was chemically synthesized and inserted into a pMIR-RePORT ${ }^{\mathrm{Tm}}$ vector (Ambion). The pMIR-RePORT ${ }^{\mathrm{Tm}} \beta$-galactosidase control vector (Ambion) was used as a reference. For the luciferase assay, 293T cells (human embryonic kidney 293 cells) were co-transfected with wild-(pMIR-MESDC1-wt) or mutanttype (pMIR-MESDC1-mt) reporter vectors and miR-508-5p mimics using Lipofectamine 2000 transfection reagent. Luciferase activity was measured $48 \mathrm{~h}$ post-transfection using a Dual-Luciferase Assay kit (Promega, Madison, WI, USA).

Cell cycle and apoptosis assays. The cells were harvested and cell cycle analysis and apoptosis quantification were performed by flow cytometry using a FAC Scan flow cytometer (Becton Dickinson Immunocytometry Systems, San Jose, CA, USA) equipped with an argon ion laser (488 nm). Cell cycle analysis was performed by flow cytometry using a propidium iodide (PI) cell cycle detection kit (Beyotime Institute of Biotechnology, Beijing, China) according to the manufacturer's 


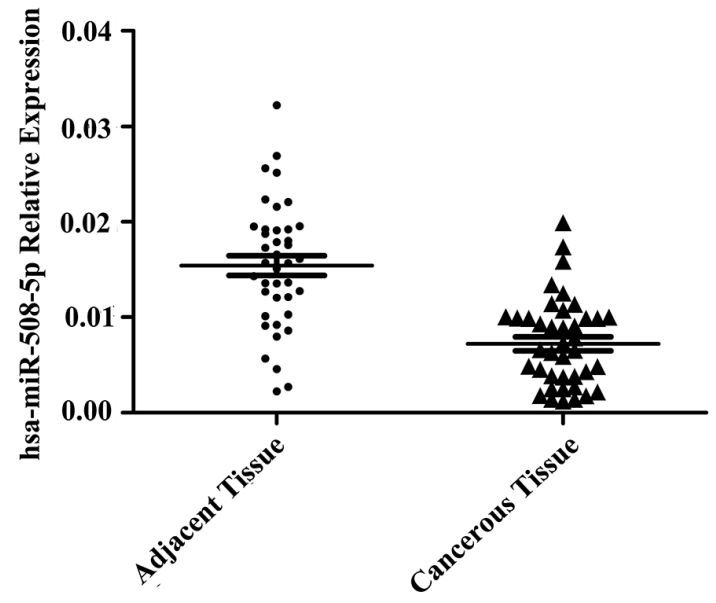

Figure 1. miR-508-5p mRNA levels in HCC tumor tissues and adjacent tissues. mRNA levels was measured in 40 paired HCC and adjacent nontumor tissues by real-time quantitative PCR. The results are expressed as the mean \pm SD. each sample was performed at least in triplicate. ${ }^{\star} P<0.01$ vs. adjacent tissues, $n=40$.

instructions. Cell apoptosis analysis was performed by flow cytometry using the fluorescein isothiocyanate Annexin V (AV) Apoptosis Detection kit I (BD Biosciences, Bedford, MA, USA) according to the manufacturer's instructions.

Cell proliferation assay. A Cell Counting Kit-8 (CCK-8; Wako Pure Chemical Industries, Osaka, Japan) was used to measure cell viability to evaluate cell proliferation. Cells treated with LV-Mock, LV-MESDC1, or LV-miR-508-5p, and cells treated with negative control or si-MESDC1 were seeded into 96-well culture plates in triplicate. At various time-points ( 0 , $12,24,36$ and $48 \mathrm{~h}), 10 \mu \mathrm{l}$ of a CCK-8 solution was added to each well in an assay plate and incubated in a $\mathrm{CO}_{2}$ incubator for $1 \mathrm{~h}$. Then, $10 \mu \mathrm{l}$ of $1 \%(\mathrm{w} / \mathrm{v})$ SDS solution was added to each well to stop the reaction and the plate was read by a microplate reader for optical density at $450 \mathrm{~nm}$ (Thermo electron Corporation, Marietta, OH, USA).

Transwell assay. Transwell chambers (Millipore-Chemicon, Billerica, MA, USA) were used for the invasion and migration assays. For the invasion assays, Matrigel-coated chambers were placed in empty wells of 24-well tissue culture plates. Cells were cultured in a serum-free medium for 24 $\mathrm{h}$, then trypsinized and suspended with a culture medium containing $0.1 \%$ bovine serum albumin at a concentration of $5 \times 10^{4}$ cells $/ \mathrm{ml}$. Then, $500 \mu \mathrm{l}$ of the cell suspension was added to each chamber and $750 \mu$ l of complete medium was added to each well. After incubation for $36 \mathrm{~h}$, the noninvading cells were removed from the upper surface of the chamber membrane using cotton-tipped swabs. The cells on the lower surface of the chamber membrane were fixed in $4 \%$ paraformaldehyde for $10 \mathrm{~min}$, stained with crystal violet, then counted after photographing the membrane through a microscope. For the migration assays, non-coated chambers were used and migrated cells on the lower surface of the chamber membrane were fixed after a 24 -h incubation, stained and photographed for further analysis.

Tumor xenograft experiments. All studies were performed in accordance with protocols approved by the Animal Use and Care Committee of Guangzhou Medical University. Athymic nude mice (BALB/c, specific pathogen-free grade, male, $\sim 16$ g, 4 week-old) were randomized into 3 groups [LV-Mock $(n=5)$, LV-MESDC1 $(n=5)$ and LV-miR-508-5p $(n=5)]$ and housed five per cage at $25^{\circ} \mathrm{C}$ under a 12 -h light/dark cycle, each performed in triplicate. All mice were fed with autoclaved mouse chow. The inoculation dosage was $1 \times 10^{7}$ HepG2 cells in $200 \mu \mathrm{l}$ of phosphate-buffered saline per mouse. Mice in the LV-Mock group were injected subcutaneously with control LV (LV-Mock), mice in the LV-MESDC1 group were injected subcutaneously with LVs overexpressing MESDC1 (LV-MESDC1) and mice in the LV-miR-508-5p group were injected subcutaneously with LVs overexpressing miR-508-5p (LV-miR-508-5p). The body weights of all of nude mice and metastatic tumors were measured every 3 days. All mice were sacrificed and the formed tumors were removed.

Statistical analysis. Data are expressed as the mean \pm standard deviation (SD). The results were analyzed by one-way analysis of variance, followed by the Student-Newman-Keuls (SNK) test and the Student's t-test using SPSS v13.0 statistical software (SPSS, Inc., Chicago, IL, USA). A two-tailed probability $(\mathrm{P})$-value $<0.05$ was considered statistically significant.

\section{Results}

Expression of miR-508-5p is downregulated in HCC tissues. It has been reported that miR-508-5p was a downregulated miRNA in the primary gastric cancer tissues. To identify whether miR-508-5p could be downregulated in liver cancer tissues, the expression of miR-508-5p was first determined in 40 paired HCC and adjacent non-tumor tissues by real-time PCR analysis. As shown in Figure 1, the expression of miR508-5p mRNA in 38 HCC tissues was lower than that in paired adjacent tissues. Our data suggest that miR-508-5p may play a critical role in the progression of HCC.

miR-508-5p inhibits cell migration, invasion and proliferation, while promotes apoptosis in vitro. To assess the role of miR-508-5p in HCC tumorigenesis, we performed cell proliferation, migration, invasion, and apoptosis assays in HCC cells transfected with empty LV vectors (LV-Mock) or human miR-508-5p overexpression LV vectors (LV-miR508-5p). The transwell assay was first used to examine the effect of miR-508-5p on HepG2 cells migration and invasion. As shown in Figure 2A, the migration and invasion rate of LV-miR-508-5p transfected HepG2 cells were significantly suppressed compared to LV-Mock transfected cells. Next, proliferative effect of miR-508-5p was confirmed by CCK-8 assay (Figure 2B), which shown that treatment with the LVmiR-508-5p vector significantly suppressed the proliferation rate of cells compared to treatment with the LV-Mock vector in the HepG 2 cells. The effects of miR-508-5p on the cell cycle 
were further examined via flow cytometry. As shown in Figure $2 \mathrm{C}$, overexpression of miR-508-5p led to a remarkable increase in the percentage of cells in the G1 phase with a concomitant decrease in the percentage of cells in the $\mathrm{S}$ phase, but has no effect on the percentage of cells in the G2/M phase compared to the control cells. We then stained the cells with AV and PI after transfection of LV-miR-508-5p or LV-Mock for apoptosis analysis. As shown in Figure 2D, with the treatment of LVmiR-508-5p, the percentage of apoptotic cells was significantly increased compared to control group. Taken together, these results suggested that miR-508-5p may play an important role in suppression of HCC carcinogenesis.

MESDC1 is a direct target of miR-508-5p. It has been shown that miRNAs performed biological functions through negatively regulating their target genes in diverse processes of life. To uncover the mechanisms by which miR-508-5p affected HCC progression, we performed bioinformatics analysis and attempted to find the potential target which might be involved in miR-508-5p mediated tumor-suppression functions. MESDC1 was identified as a potential target of miR-508-5p, utilizing a combination of TargetScan, miRanda, RNA22, miRDB, miR-Gen, PITA, eMBL-eBI, starBase, PicTar, RNAhybrid, and miRBase. MiRNAs decreased the mRNA level of target genes through binding to the $3^{\prime}$-UTR of the target gene. To confirm the direct binding between miR-508-5p and MESDC1 3'-UTR, the dual-luciferase assay was performed. As shown in Figure 3A, human MESDC1 mRNA contains one puta-
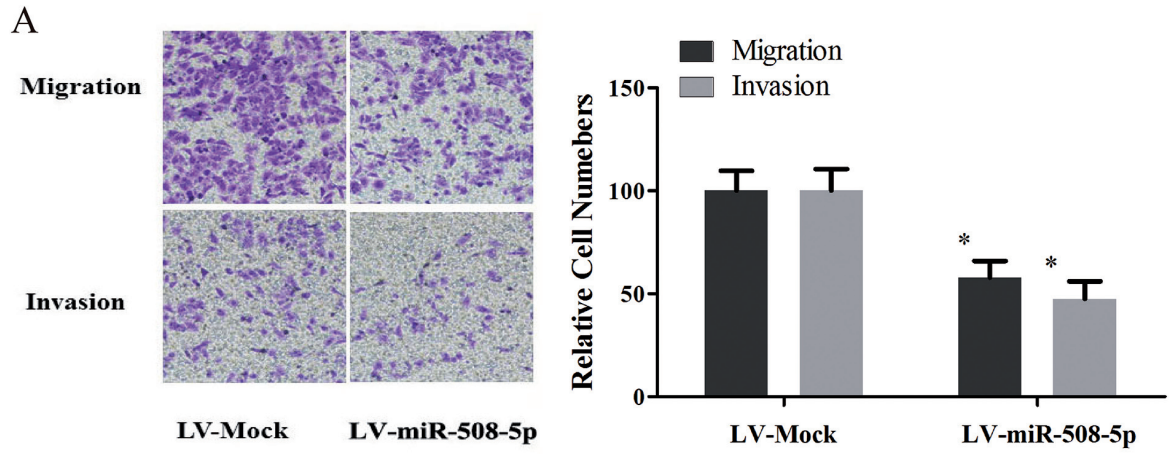

B

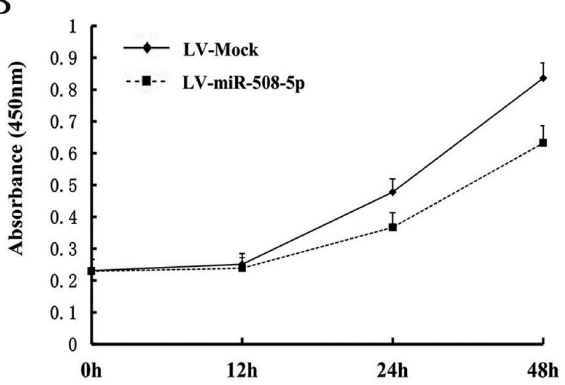

$\mathrm{C}$
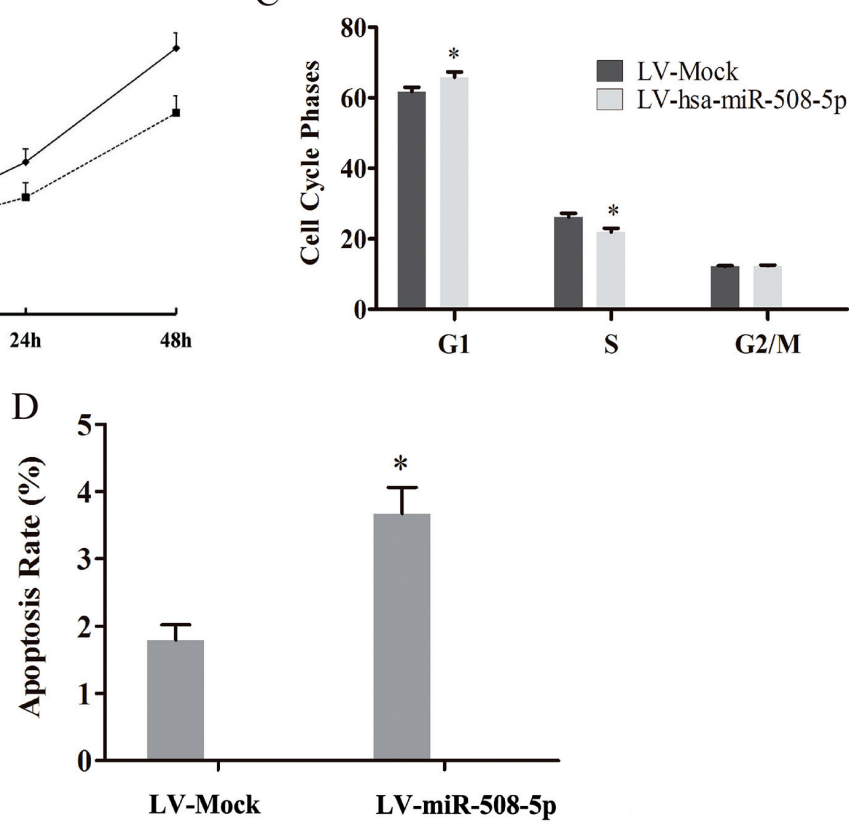

Figure 2. Impact of miR-508-5p on HepG2 cells invasion, migration, proliferation and apoptosis. HepG2 cells were treated as indicated and (A) Transwell assays were performed to assess migration and invasion by analyzing the number of cells on the Transwell membrane. Cells in five randomlyselected fields were counted. Representative images are shown (magnification, 40x). (B) The CCK-8 proliferation assay was performed. (C) The cell cycle distribution was assessed via flow cytometry. (D) The proportion of apoptotic cells was assessed by flow cytometry $(50,000$ cells counted). AV+PI - were defined as early apoptosis. The results are expressed as the mean \pm SD of three independent experiments, each performed in triplicate. ${ }^{\star} P<0.05 \mathrm{vs.} \mathrm{LV-Mock}$ group. AV, Annexin V; PI, propidium iodide, $n=5$. 
tive complementary sequence to miR-508-5p. Meanwhile, luciferase activity was significantly decreased in $293 \mathrm{~T}$ cells co-transfected with wild-type (pMIR-MESDC1-wt) reporter vectors and miR-508-5p. Mutation of binding site completely reversed the effect of miR-508-5p. Western blot analysis confirmed that mimics of miR-508-5p were able to suppress endogenous MESDC1 protein expression (Figure 3B). To further elucidate the relationship between miR-508-5p and MESDC1 in HCC, we detected the MESDC1 expression in the same 40 paired HCC and adjacent tissues using real-time PCR. We found that the level of MESDC1 was increased in the HCC tissues compared to adjacent non-tumor tissues (Figure 3C). Thus, our findings indicate that MESDC1 is a potential target of miR-508-5p in HCC.

MESDC1 promotes cell invasion, migration and proliferation, while inhibits apoptosis in vitro. Since MESDC1 is the target gene of miR-508-5p, we therefore asked if miR508-5p exerted its tumor-suppressive functions through targeting MESDC1. We then transfected HepG2 cells with an adenovirus expressing MESDC1 (LV-MESDC1) or control adenovirus (LV-Mock). As shown in Figure 4A, the protein expression of MESDC1 was significantly increased after overexpression of MESDC1 gene in LV-MESDC1-transfected cells. We further assessed the effects of MESDC1 on HCC cells migration and invasion, which were the key determinants of malignant progression and metastasis. As shown in Figure $4 \mathrm{~B}$, transwell assay indicated that LV-MESDC1-transfected cells exhibited remarkable increase of migration and invasion abilities compared to LV-Mock - transfected cells. In addition, we investigated the effects of MESDC1 on the HepG2 cells survival or proliferation in vitro using the CCK-8 assay, which showed that LV-MESDC1 promoted the proliferation of HepG2 cells (Figure 4C). Consistently, compared to the control cells, LV-MESDC1-transfected cells exhibited an increase in the percentage of cells in the G1 phase, a decrease in the percentage of cells in the $S$ phase, and no change in the percentage of cells in the G2/M phase (Figure 4D). Moreover, LV-MESDC1 significantly suppressed the HepG2 cells apoptosis rate (Figure 4E). Next, we further checked these phenotypes by knocking down MESDC1. As expected, the results showed that si-MESDC1 markedly suppressed tumor cell proliferation and promoted cell apoptosis (Figure $4 \mathrm{~F}$ and $4 \mathrm{G})$, respectively. Moreover, knocking down of MESDC1
A

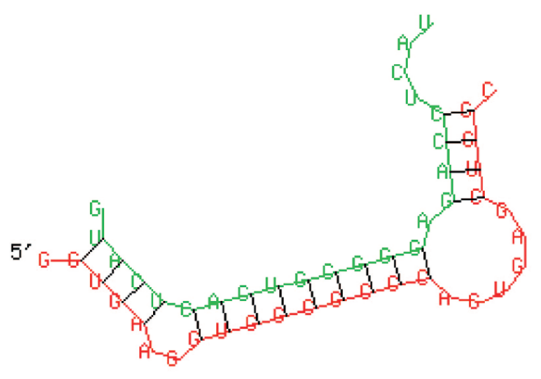

mfe: $-30.3 \mathrm{kcal} / \mathrm{mol}$

C

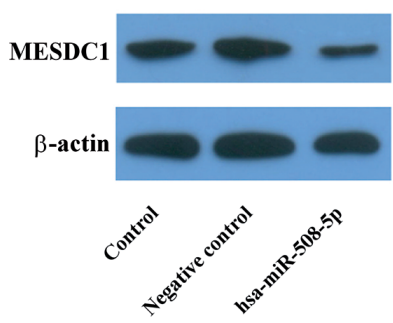

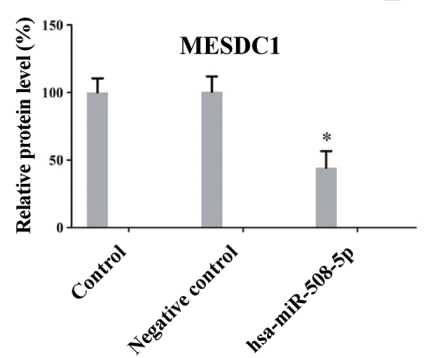

B

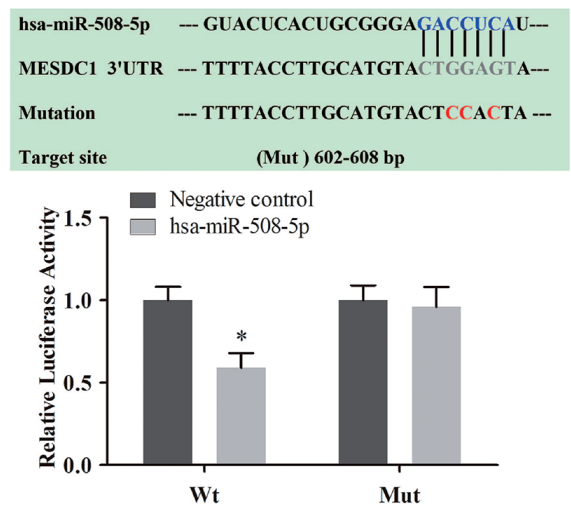

D

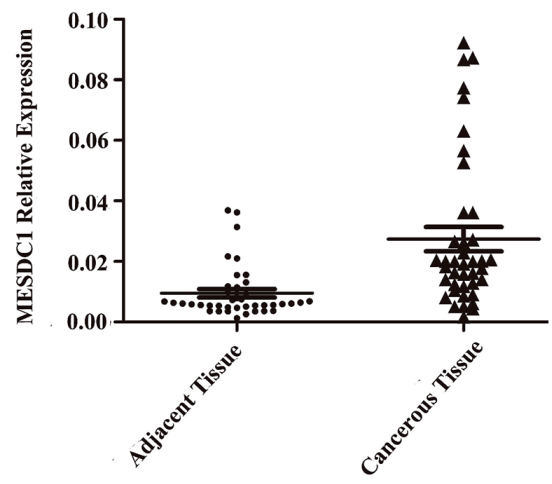

Figure 3. (A) Sequence alignment of the miR-508-5p mature sequence with the binding sites of the MESDC1 3'UTR (Microcosm Targets). (B) Putative miR-508-5p-binding site in MESDC1 3'UTR and the corresponding binding site were shown. (C) The protein level of miR-508-5p was analyzed by western blotting after treatment with miR-508-5p mimics or negative control. The results are expressed as the mean \pm SD of three independent experiments, each performed in triplicate. (D) RT-PCR analysis of MESDC1 mRNA expression in HCC tumor tissues and adjacent tissues. The results are expressed as the mean \pm SD of three independent experiments, each performed in triplicate. ${ }^{\star} P<0.05$ vs. negative control group, $\mathrm{n}=5$. 

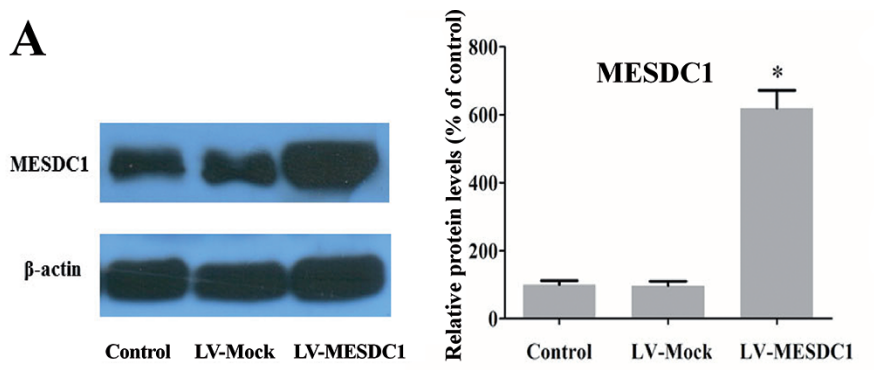

B
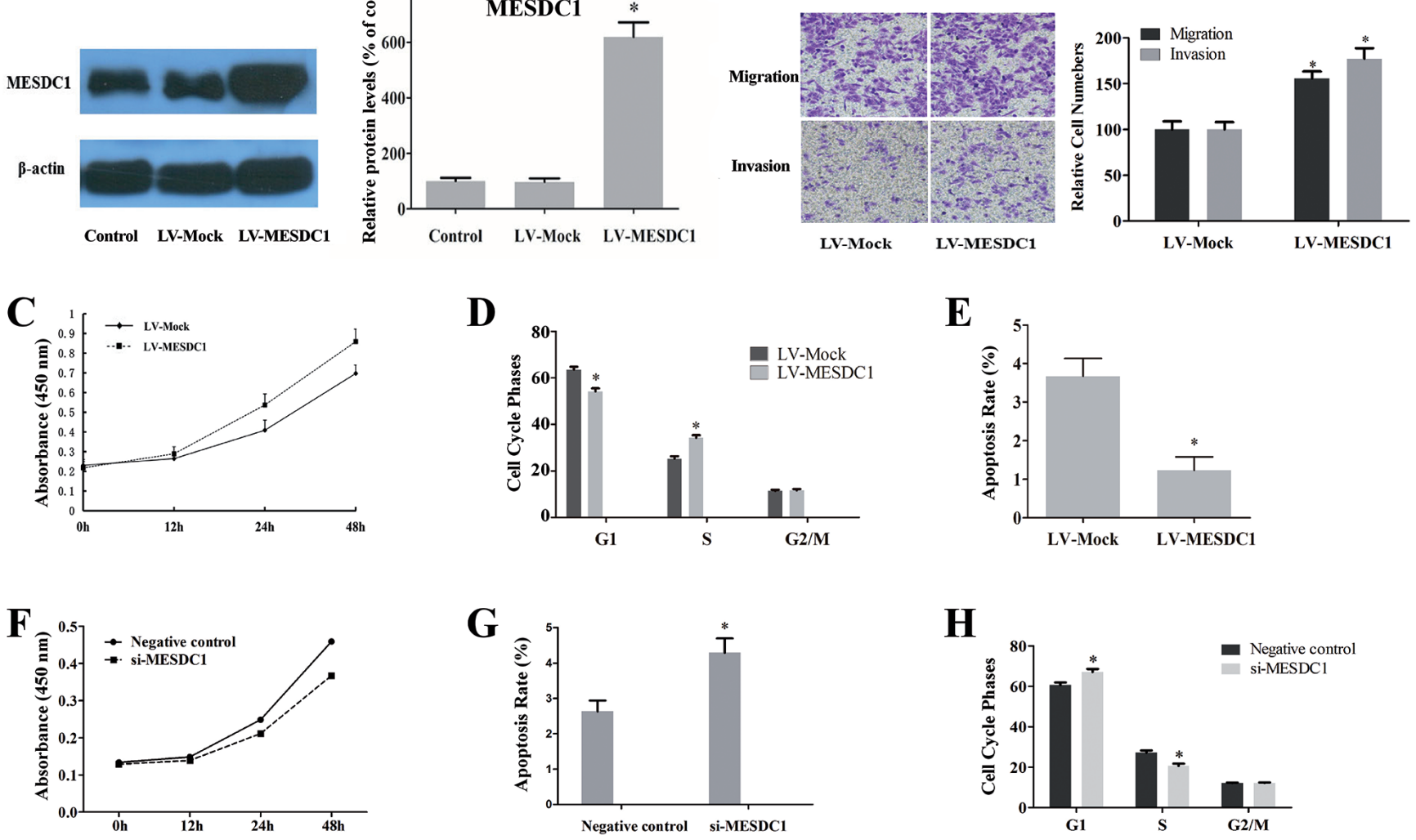

Figure 4. Impact of MESDC1 on HepG2 cells invasion, migration, proliferation and apoptosis. HepG2 cells were treated as indicated and (A) MESDC1 protein expression was measured by western blot analysis. (B) Transwell assay were performed to assess migration and invasion by analyzing the number of cells on the Transwell membrane. Cells in five randomlyselected fields were counted. Representative images are shown (magnification, 40x). (C and F) The CCK-8 proliferation assay was performed. (D and H) The cell cycle distribution was assessed via flow cytometry. (E and G) The proportion of apoptotic cells was assessed by flow cytometry (50,000 cells counted). AV+PI - were defined as early apoptosis. The results are expressed as the mean \pm SD of three independent experiments, each performed in triplicate. ${ }^{\star} P<0.05$ vs. LV-Mock group. AV, Annexin V; PI, propidium iodide, $n=5$.

A

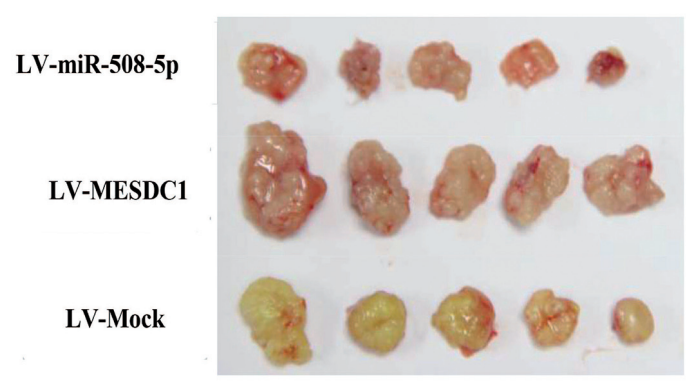

B

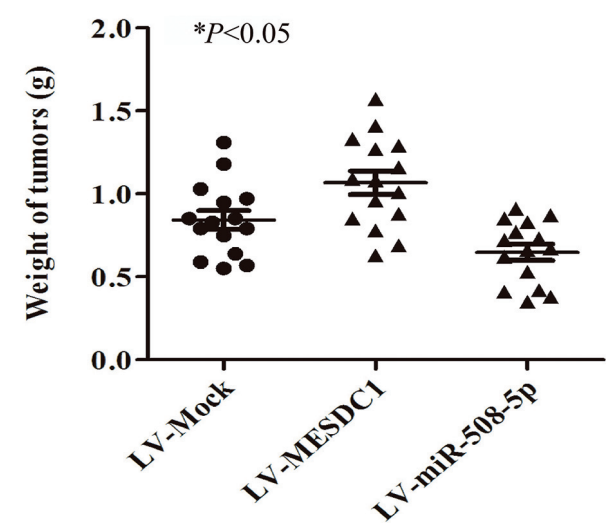

Figure 5. Effects of miR-508-5p and MESDC1 on tumor growth of xenograft model. HepG2 cells transfected with LV-miR-508-5p, LV-MESDC1 or LV-Mock were injected subcutaneously into the nude mice. (A) Representative tumors were removed and photographed (n=5). (B) The weights of tumors in all nude mice were measured. The results are expressed as the mean \pm SD of three independent experiments, each performed in triplicate. ${ }^{\star} P<0.05$ vs. $\mathbf{L V}$-Mock group. 
resulted in a remarkable increase in the percentage of cells in the G1 phase with a concomitant decrease in the percentage of cells in the $S$ phase, but has no effect on the percentage of cells in the G2/M phase compared to the control cells (Figure $4 \mathrm{H})$. These results provided evidences that MESDC1 was involved in miR-508-5p mediated suppression of HCC cells proliferation and metastasis.

miR-508-5p suppresses HCC tumorigenesis, while MESDC1 promotes the HCC tumorigenesis in vivo. To assess the role of miR-508-5p and MESDC1 in HCC tumorigenesis in vivo, HepG2 cells transfected with an adenovirus expressing MESDC1 (LV-MESDC1), miR-508-5p (LV-miR-508-5p) or control adenovirus (LV-Mock) were injected subcutaneously into nude mice. Four weeks after the subcutaneous injections, the tumors were removed and weighed. In vivo experiments showed that tumor growth was promoted in the mice injected with LV-MESDC1-treated HepG2 cells and reduced in the mice injected with the LV-miR-508-5p-treated HepG2 cells compared to mice injected with LV-Mock-treated HepG2 cells (Figure 5A and 5B). These data indicate that MESDC1 promotes HCC tumorigenesis, while miR-508-5p acts as a tumor suppressor gene in HCC.

\section{Discussion}

Almost $85 \%$ of new HCC cases are diagnosed each year worldwide with disease burden highest in developing countries $[4,21]$. Generally, due to lack of reliable markers for early diagnosis, tumor recurrence, resistance to treatment, acceleration of cell proliferation and ultimately metastasis, patients with HCC often exhibited poor prognosis and clinical outcome [22]. The progression of HCC is a complicated process that involves the dysregulation of multiple genes that are critical for cell biological processes. Recent studies have demonstrated that aberrant expression of miRNAs is closely related to occurrence, diagnosis, development, and staging of HCC [23]. However, miRNAs that modulate the carcinogenesis and progression of HCC have not been precisely explored. MiR-508-5p has been reported as a downregulated miRNA in gastric cancer tissues which influenced drug resistance $[17,18]$. However, little is known about the function of miR-508-5p in HCC. In this research, we identified that miR-508-5p functioned as a tumor suppressor in the progression of $\mathrm{HCC}$ by negative regulation of the target MESDC1 expression.

Aberrant expression of miR-508-5p has been reported in diverse biological processes. High expression of miR-508-5p in endothelial progenitor cells was associated with an increased risk of cardiovascular death and decreased survival rate [24]. In addition, miR-508-5p could directly target the 3'-UTR regions of $A B C B 1$ and ZNRD1, resulting in enhancement of cancer cell resistance to multiple chemotherapeutics in vitro and in vivo [18]. These researches indicate that aberrant miR-508-5p expression may play an essential role in tumors pathogenesis. In our study, we found that miR-508-5p
mRNA levels were downregulated in HCC tissues compared with paired adjacent non-tumor tissues. Previous reports showed that differential expression of miR-508-5p was associated with paclitaxel-induced apoptosis in hepatocellular carcinoma cells [25]. To further determine the role of miR-508 $-5 p$ in the progression of HCC, HepG2 cells transfection with LV-miR-508-5p was performed. Furthermore, our functional studies demonstrated that overexpression of miR-508-5p significantly suppressed HepG2 cells migration, invasion and proliferation, while promoting apoptosis. These results indicate that miR-508-5p could act as a tumor suppressor in carcinogenesis of HCC.

Using bioinformatics and luciferase reporter assays, we demonstrated that MESDC1 is a direct downstream target of miR-508-5p. Moreover, overexpression of miR-508-5p reduced MESDC1 protein levels in HepG2 cells. MESDC1 has been reported as an oncogene in bladder cancer (BC) cell lines by inducing cell viability, migration and invasion and suppressing apoptosis [19]. However, the expression and mechanism of MESDC1 in HCC is still unidentified. In the present study, MESDC1 was increased in the HCC tissues compared to that in adjacent non-tumor tissues. In addition, over-expressed MESDC1 promoted HCC invasion, migration and proliferation, while suppressing apoptosis in vitro. The results showed that MESDC1 could promote tumor cell proliferation, inhibit apoptosis and promote tumor cell growth in vitro. Consistent with our in vitro findings, orthotropic xenograft experiments demonstrated that overexpression of miR-508-5p inhibited tumor growth in xenograft model, while overexpression of MESDC1 promoted tumor growth in xenograft model, suggesting that MESDC1 could promote HCC growth and miR-508-5p is a tumor-suppressive miRNA in HCC. These data confirmed that miR-508-5p regulated biological behavior of HCC by directly targeting MESDC1. However, further comprehensive investigations are needed to uncover the mechanism of MESDC1 in HCC.

In summary, we present evidence that miR-508-5p could act as a tumor-suppressive miRNA by directly downregulating MESDC1 expression in HCC progression. Our in vitro and in vivo studies suggested that restoration of miR-508-5p and inhibition of MESDC1 may provide more effective therapeutic strategy for HCC development.

Acknowledgments: This work was supported by Guangdong Medical Research foundation (No. B2012311) and Guangzhou City Health Bureau's Medical Research foundation (No. 20121A011099, 201102A213229).

\section{References}

[1] NAUlT JC, DE REYNIES A, VillanueVA A, CALDERARO J, REBOUISSOU S et al. A hepatocellular carcinoma 5-gene score associated with survival of patients after liver resection. Gastroenterology 2013; 145: 176-187. http://dx.doi.org/10.1053/j.gastro.2013.03.051 
[2] PARIKH S, HYMAN D. Hepatocellular cancer: a guide for the internist. Am J Med 2007; 120: 194-202. http://dx.doi. org/10.1016/j.amjmed.2006.11.020

[3] OKUDA K. Hepatocellular carcinoma. J Hepatol 2000; 32 (1 Suppl): 225-237. http://dx.doi.org/10.1016/S0168-8278(00)80428-6

[4] WAGHRAY A, MURALI AR, MENON KN. Hepatocellular carcinoma: From diagnosis to treatment. World J Hepatol 2015; 7 : 1020-1029. http://dx.doi.org/10.4254/wjh.v7.i8.1020

[5] HONG Y, HUANG J. Autoantibodies against tumor-associated antigens for detection of hepatocellular carcinoma. World J Hepatol 2015; 7: 1581-1585. http://dx.doi.org/10.4254/wjh. v7.i11.1581

[6] HAMMOND SM. An overview of microRNAs. Adv Drug Deliv Rev 2015; 87: 3-14. http://dx.doi.org/10.1016/j. addr.2015.05.001

[7] SU Z, YANG Z, XU Y, CHEN Y, YU Q. MicroRNAs in apoptosis, autophagy and necroptosis. Oncotarget 2015; 6: 8474-8490. http://dx.doi.org/10.18632/oncotarget.3523

[8] SCHENA FP, SALLUSTIO F, SERINO G. microRNAs in glomerular diseases from pathophysiology to potential treatment target. Clin Sci (Lond.) 2015; 128: 775-788. http://dx.doi. org/10.1042/CS20140733

[9] WANG J, DU Y, LIU X, CHO WC, YANG Y. MicroRNAs as Regulator of Signaling Networks in Metastatic Colon Cancer. Biomed Res Int 2015: 823620.

[1] WANG J, CHEN J, SEN S. microRNA as Biomarkers and Diagnostics. J Cell Physiol 2016; 231: 25-30. http://dx.doi. org/10.1002/jcp.25056

[11] KONTOMANOLIS EN, KOUKOURAKIS MI. MicroRNA: the potential regulator of endometrial carcinogenesis. Microrna 2015; 4:18-25. http://dx.doi.org/10.2174/22115366046661507 10094418

[12] BAI Y, LI J, LI J, LIU Y, ZHANG B. MiR-615 inhibited cell proliferation and cell cycle of human breast cancer cells by suppressing of AKT2 expression. Int J Clin Exp Med 2015; 8: 3801-3808.

[13] ZHANG T, WANG Q, ZHAO D, CUI Y, CAO B et al. The oncogenetic role of microRNA-31 as a potential biomarker in oesophageal squamous cell carcinoma. Clin Sci (Lond) 2011; 121: 437-447. http://dx.doi.org/10.1042/CS20110207

[14] LI D, LIU Y, LI H, PENG JJ, TAN Y et al. MicroRNA-1 promotes apoptosis of hepatocarcinoma cells by targeting apoptosis inhibitor-5 (API-5). FEBS Lett 2015; 589: 68-76. http://dx.doi.org/10.1016/j.febslet.2014.11.025

[15] ZHAI J, QU S, LI X, ZHONG J, CHEN X et al. miR-129 suppresses tumor cell growth and invasion by targeting
PAK5 in hepatocellular carcinoma. Biochem Biophys Res Commun 2015; 464: 161-167. http://dx.doi.org/10.1016/j. bbrc.2015.06.108

[16] SUN X, LIU Y, LI M, WANG M, WANG Y. Involvement of miR-485-5p in hepatocellular carcinoma progression targeting EMMPRIN. Biomed Pharmacother 2015; 72: 58-65. http://dx.doi.org/10.1016/j.biopha.2015.04.008

[17] WANG Z, WANG J, YANG Y, HAO B, WANG R et al. Loss of has-miR-337-3p expression is associated with lymph node metastasis of human gastric cancer. J Exp Clin Cancer Res 2013; 32: 76. http://dx.doi.org/10.1186/1756-9966-32-76

[18] SHANG Y, ZHANG Z, LIU Z, FENG B, REN G, LI K et al. miR-508-5p regulates multidrug resistance of gastric cancer by targeting ABCB1 and ZNRD1. Oncogene 2014; 33: 3267-3276. http://dx.doi.org/10.1038/onc.2013.297

[19] TATARANO S, CHIYOMARU T, KAWAKAMI K, ENOKIDA $\mathrm{H}$, YOSHINO $\mathrm{H}$ et al. Novel oncogenic function of mesoderm development candidate 1 and its regulation by MiR-574-3p in bladder cancer cell lines. Int J Oncol 2014; 40: 951-959.

[20] XU H, HU YW, ZHAO JY, HU XM, LI SF, WANG YC et al. MicroRNA-195-5p acts as an anti-oncogene by targeting PHF19 in hepatocellular carcinoma. Oncol Rep 2015; 34: 175-182. http://dx.doi.org/10.3892/or.2015.3957

[21] LYRA-GONZALEZI, FLORES-FONG LE, GONZALEZ-GARCIA I, MEDINA-PRECIADO D, ARMENDARIZ-BORUNDA J. MicroRNAs dysregulation in hepatocellular carcinoma: Insights in genomic medicine. World J Hepatol 2015; 7 : 1530 1540. http://dx.doi.org/10.4254/wjh.v7.i11.1530

[22] GONG R, SUN D, ZHONG X, SUN Y, LI L. MEK1 expression and its relationship with clinical pathological features in hepatocellular carcinoma. Int J Clin Exp Med 2015; 8: 4087-4093.

[23] GIORDANO S, COLUMBANO A. MicroRNAs: new tools for diagnosis, prognosis, and therapy in hepatocellular carcinoma? Hepatology 2013; 57: 840-847. http://dx.doi. org/10.1002/hep.26095

[24] QIANG L, HONG L, NINGFU W, HUAIHONG C, JING W. Expression of miR-126 and miR-508-5p in endothelial progenitor cells is associated with the prognosis of chronic heart failure patients. Int J Cardiol 2013; 168: 2082-2088. http:// dx.doi.org/10.1016/j.ijcard.2013.01.160

[25] YAN H, WANG S, YU H, ZHU J, CHEN C. Molecular pathways and functional analysis of miRNA expression associated with paclitaxel-induced apoptosis in hepatocellular carcinoma cells. Pharmacology 2013; 92: 167-174. http:// dx.doi.org/10.1159/000354585 